\title{
Análisis del desempeño académico de estudiantes de una institución de educación superior en Ecuador, antes y durante la pandemia Analysis of the academic performance of students from a higher education institution in Ecuador, before and during the pandemic
}

\author{
Tarquino Sánchez-Almeida ${ }^{1}$, David Naranjo ${ }^{2}$, Jessica Reina ${ }^{2}$ \\ Tarquino.sanchez@epn.edu.ec,david.naranjo@epn.edu.ec,jesslore_1@hotmail.com \\ ${ }^{1}$ Departamento de Electrónica, \\ Telecomunicaciones y Redes de la Información. \\ Escuela Politécnica Nacional \\ Quito, Ecuador \\ ${ }^{2}$ Grupo de Investigación Educativa INEDU-EPN \\ Escuela Politécnica Nacional \\ Quito, Ecuador
}

\begin{abstract}
Resumen- La pandemia provocada por el coronavirus SARS-CoV-2 cambió drásticamente las actividades diarias a nivel mundial en el ámbito de la educación superior, muchas universidades debieron realizar sus funciones en modalidad virtual. La Escuela Politécnica Nacional es una universidad ecuatoriana, cuya modalidad de estudio se mantuvo presencial por más de 150 años de historia. Sin embargo, desde la pandemia dicha universidad tuvo que adaptar todas sus clases a la modalidad virtual. En este estudio, realizamos un análisis comparativo del desempeño académico entre los estudiantes que ingresaron al curso de nivelación de la Institución de Educación Superior durante la pandemia y los que lo hicieron antes de la pandemia. Para ello, analizamos la información de 9.267 estudiantes que ingresaron a la institución por primera vez entre 2017 y 2020. Realizamos un Análisis de Varianza cuyo factor fue la modalidad de estudio, y luego realizamos una prueba post hoc de Tukey para identificar grupos homogéneos. Los resultados mostraron que los estudiantes que ingresaron al curso de nivelación en modo virtual presentaron un desempeño académico significativamente superior al desempeño académico histórico de los estudiantes que tuvieron clases presenciales. Sin embargo, los resultados no necesariamente indican que los estudiantes de ese período y modalidad hayan obtenido más conocimientos. Estos hallazgos coinciden con algunos autores que establecen que el desempeño académico de los estudiantes universitarios no es un referente para la evaluación cognitiva en toda su magnitud, ya que este proceso provoca el etiquetado de ellos, los sujetos y los propios docentes, ubicándolos en un rango de evaluación. que pueden determinar la capacidad, eficacia y calidad del proceso de enseñanza-aprendizaje.
\end{abstract}

Palabras clave: Rendimiento académico, Coronavirus, Análisis de Datos

Abstract- The coronavirus SARS-CoV-2 pandemic dramatically changed daily activities globally. In the field of higher education, many universities had to carry out their functions in virtual mode. The Escuela Politécnica Nacional is an Ecuadorian university, the study modality of which stayed face-to-face in more than 150 years of history. However, since the pandemic, said university had to adapt all its classes to the virtual modality. In this study, we conducted a comparative analysis of academic performance between students who entered to the university leveling course during the pandemic and those who did so before the pandemic. To do this, we analyzed the information of 9,267 students who entered the institution for the first time between 2017 and 2020. We carried out a Variance Analysis the factor of which was the study modality, and then we carried out a Tukey post hoc test to identify homogeneous groups. The results showed that the students who entered the leveling course in virtual mode presented an academic performance significantly higher than the historical academic performance of the students who had face-to-face classes. Nevertheless, results do not necessarily indicate that the students of that period and modality have obtained more knowledge. These findings coincide with some authors who establish that the academic performance of university students is not a reference for cognitive evaluation in all its magnitude, since this process causes the labeling of them, the subjects, and the teachers themselves, placing them in a range evaluation that can determine the capacity, effectiveness, and quality of the teaching-learning process.

\section{Keywords: Academic performance, Covid -19, Data Analysis}

\section{INTRODUCCIÓN}

El 30 de enero de 2020, la Organización Mundial de la Salud declaró la pandemia de COVID-19 una emergencia de Salud Pública, que hasta la fecha ha afectado a un gran número de personas en todo el mundo (Organización Mundial de la Salud, 2020). El nuevo coronavirus SARS-CoV-2, responsable de la enfermedad COVID-19, se ha extendido por todo el planeta, sumando más de 3 millones de muertes y cerca de 146,4 millones de infectados, registrados hasta el 25 de mayo de 2021 (Universidad Johns Hopkins, 2021). La agresividad del virus y su fácil transmisión ha obligado a las actividades que se desarrollan en la sociedad a trasladarse a un espacio que minimiza la interacción cara a cara. En el caso de la educación, según datos de la Organización de las Naciones Unidas para la Educación, la Ciencia y la Cultura (UNESCO), a mediados de mayo de 2020, en la región de América Latina y el Caribe, más de 160 millones de estudiantes de todos los niveles educativos, habían dejado de tener clases presenciales en la escuela (CEPAL-UNESCO, 2020).

La Comisión Económica para América Latina y el Caribe (CEPAL) (2020) indicó que, incluso antes de enfrentar la pandemia, la situación social en la región se estaba deteriorando, debido al aumento de las tasas de pobreza y pobreza extrema, la persistencia de las desigualdades y el crecimiento social. En este sentido, el impacto de la pandemia 
ha tenido importantes efectos negativos en los diferentes sectores sociales, en particular la salud y la educación, así como en el empleo y la lucha contra la pobreza.

En este contexto, el sistema educativo atraviesa una situación crítica, producto de la pandemia COVID-19, que ha afectado particularmente a los estudiantes en la modalidad de estudio presencial. Aun así, las instituciones educativas han desarrollado estrategias para dar continuidad a los programas académicos a través de un cambio de modalidad hacia la educación virtual, que, en paralelo, ha presentado inconvenientes como la falta de recursos, especialmente tecnológicos, por parte de los estudiantes, lo que refleja las limitaciones económicas que enfrentan y que limitan su acceso a la conectividad y los medios digitales a sus hogares. Esto, junto con el aislamiento social obligatorio como medida preventiva, influye directamente en el desempeño de los estudiantes en los entornos virtuales, en el ámbito emocional y en el entorno familiar de los estudiantes (D'Amelio et al., 2020).

Sin embargo, es destacable que en muchos casos la enseñanza-aprendizaje virtual que ofrecen la mayoría de Instituciones de Educación Superior (IES), ha permitido a los estudiantes mejorar sus habilidades y competencias académicas, creando las bases para un constante crecimiento personal, permitiendo al mismo tiempo promover la autogestión, la reflexión crítica y la autonomía del alumno en pos de su desarrollo integral, preparándolo para afrontar la vida, con pensamiento constructivo y positivo, tendiente al éxito en su formación profesional, personal y familiar (Parada \& Pérez, 2014).

Una investigación similar en (Benítez \& Cabrera, 2021) determinó que los promedios de las notas obtenidas de la cohorte mayo 2020 - 2021 con modalidad virtual debido a la pandemia, resultaron mejores en un $52.52 \%$ en comparación con la cohorte anterior, la cual fue de manera presencial. Sin embargo, la percepción de los estudiantes mostró que las conferencias, trabajos colaborativos, talleres, laboratorios y casos clínicos eran mejor orientados y con mayor participación de los estudiantes en forma presencial, por lo que una ventaja es el ahorro de tiempo al recibir las clases en casa y la desventaja es que se tuvieron falencias en el nivel practico como se ha mencionado.

El incremento en el uso de tecnologías digitales fue fundamental en la educación virtual, la cual fue intervenida por estrategias basadas en sistemas programados para enfrentar realidades complejas como las evaluaciones académicas, pero que deben fortalecerse especialmente aquellas que corresponden al entorno tecnológico institucional, con la accesibilidad. Y uso de Internet por parte de los estudiantes (Paredes Chacín et al., 2020). Así, el trabajo que realizan las IES se enmarca en uno de los seis principios rectores para poder enfrentar la crisis del COVID-19 en la educación superior, como lo señalan la UNESCO y el Instituto Internacional de Educación Superior en América Latina y el Caribe (IESALC), que hace referencia a que "la reanudación de las actividades presenciales en las IES debe verse como una oportunidad para repensar y, en la medida de lo posible, rediseñar los procesos de enseñanza y aprendizaje, aprovechando las lecciones que el uso intensivo de tecnología puede haber supuesto, prestando especial atención a la equidad y la inclusión" (Ordorika Imanol, 2020).
Si bien las universidades y los estudiantes tienen clara la importancia de la formación virtual, las facilidades y la flexibilidad que permite esta modalidad, debemos ser conscientes de que no todos los campos y carreras pueden trasladar todas sus asignaturas al mundo virtual. Estas carreras que presentan inconvenientes con lo virtual son principalmente carreras técnicas, científicas o sanitarias, en las que el aprendizaje práctico es el pilar de la docencia y la clave para poder ejercerlas. Quizás en los dos primeros años de estas carreras se pueda trabajar virtualmente, ya que en las carreras técnicas las prácticas de especialización son mínimas. Es posible que el alumno pueda contar con material en línea para saber cómo realizar diferentes trámites; pero al final se necesita práctica para aprender a hacerlo y perfeccionarlo. También es cierto que aún quedan algunos detalles por aclarar en la formación virtual, especialmente en relación a la experiencia del alumno y la interacción con otros participantes, en este momento es difícil trasladar la experiencia práctica de aprendizaje a las aulas virtuales (Universia.net, 2020). En este contexto, en el caso particular de la Escuela Politécnica Nacional (EPN), en Quito-Ecuador, la modalidad de estudio siempre ha sido presencial, hasta el momento en que comenzó la pandemia, donde drásticamente el semestre del primer período de 2020 se desarrolló de forma virtual. La EPN ofrece títulos en ciencias físicas y matemáticas, economía, ingeniería técnica y tecnológica, cuya transformación virtual no se había implementado en su totalidad. Para acceder a las carreras mencionadas, los nuevos estudiantes deben superar el curso regular de nivelación desarrollado por el Departamento de Formación Básica de la misma universidad. El curso de nivelación EPN incluye en su programa académico las asignaturas de: Matemáticas, Geometría, Física, Química, Lenguaje y Comunicación, las cuales deben ser aprobadas íntegramente por los estudiantes, para ser promovidas al primer nivel de la carrera que hayan seleccionado para su formación profesional, según lo establecido en el Reglamento del Régimen Académico (CES., 2019). La modalidad virtual representó un riesgo, pero a la vez un desafío, para las autoridades, docentes y estudiantes de la institución, ya que no hubo una intervención previa, adecuada y oportuna para los ambientes virtuales de aprendizaje, debiendo adecuarse, de manera repentina $\mathrm{y}$ estratégica, la contenidos y actividades que puedan encajar en las aulas virtuales, proporcionando los espacios virtuales necesarios para la interacción entre profesores y alumnos.

Esta investigación tiene como objetivo realizar un análisis comparativo del desempeño académico de los estudiantes que se matricularon en el curso de nivelación EPN antes y durante la pandemia COVID-19, a través de los resultados académicos obtenidos al final de cada período de estudio, y así determinar el potencial efectos del cambio de modalidad presencial a virtual en los primeros años de la universidad

\section{CONTEXTO}

El análisis de información se realiza sobre un total de 9.267 que ingresaron al curso de nivelación de la EPN, entre 2017 y 2020. Durante cada año escolar, la institución abre dos trimestres denominados A y B, siendo el trimestre 2020-A el primero que fue Totalmente desarrollado en línea, debido a la emergencia sanitaria provocada por COVID-19. La Tabla 1 muestra la distribución de los participantes del estudio.

El método utilizado fue un análisis exploratorio y descriptivo de los datos obtenidos antes de la pandemia (2017-A hasta 
2019-B), al cual se practicó el análisis de varianza ANOVA y luego la prueba post hoc de Tukey para identificar grupos homogéneos, y, compararlos con el rendimiento académico de los estudiantes matriculados por asignatura en el periodo académico 2020-A en modalidad virtual. En la cual, la prueba de Tukey provee un nivel de significancia global de $\alpha$ cuando los tamaños de las muestras son iguales y de $\alpha$ a lo sumo, cuando no son iguales.

Tabla 1: Distribución de las participantes en el estudio.

\begin{tabular}{ccc}
\hline Período & Modalidad & $\begin{array}{c}\text { Número de } \\
\text { estudiantes }\end{array}$ \\
\hline 2017-A & Presencial & 1505 \\
2017-B & Presencial & 1373 \\
2018-A & Presencial & 1344 \\
2018-B & Presencial & 1413 \\
2019-A & Presencial & 1237 \\
2019-B & Presencial & 1071 \\
2020-A & Virtual & 1324 \\
\hline \multicolumn{3}{c}{ Total } \\
\hline
\end{tabular}

\section{A. Mediciones}

Se determinó la puntuación final sobre 40 puntos, obtenida por los estudiantes en cada una de las asignaturas de la asignatura de nivelación - Matemáticas, Geometría, Física, Química y Lenguaje y Comunicación - en cada uno de los semestres académicos; la nota refleja los resultados del trabajo de los estudiantes durante el semestre, a través de la entrega de trabajos y tareas, así como la realización de pruebas y exámenes.

Asimismo, los alumnos que superaron la asignatura de nivelación fueron identificados con base en su calificación final de 40 puntos, de acuerdo con lo establecido en el Reglamento de Régimen Académico de la EPN e incluirá el número de subsecciones necesarias en cada sección.

\section{B. Procedimiento}

La información utilizada en este estudio se obtuvo de las bases de datos de la Dirección de Gestión de la Información y Procesos de la EPN. Los datos de todos los estudiantes considerados en la investigación se manejaron con base a acuerdos institucionales de confidencialidad, los cuales contemplan el procesamiento de la información con fines puramente investigativos. En este estudio, solo se presenta información totalizada y anónima.

\section{DESCRIPCIÓN}

Se realizó un análisis descriptivo del porcentaje de aprobación del curso de nivelación, en función de la modalidad presencial y virtual, así como una prueba de independencia entre el período de admisión al curso de nivelación y la aprobación. Además, se realizó un análisis de la varianza de un factor de la nota final, de cada una de las asignaturas del curso de nivelación, entre cada semestre académico. Finalmente, se realizó un post test hoc Tukey para identificar grupos homogéneos. Todos los análisis se realizaron con un nivel de significancia del 5\% y se realizaron en SPSS V22.

\section{RESUltados}

En la Figura 1 se presenta el porcentaje de estudiantes que aprobaron y reprobaron el curso de recuperación, según la modalidad de estudios. Se aprecia que el porcentaje de aprobación en la modalidad virtual es prácticamente el doble que en la modalidad presencial.

Por otro lado, la Figura 2. Muestra los resultados de la prueba de independencia realizada entre el período de ingreso al curso de nivelación y la aprobación. Se puede observar que a un nivel de significancia del 5\%, la aprobación no es independiente del período de inicio del curso de nivelación, y se observa que el mayor porcentaje histórico de aprobación, correspondiente al $25.2 \%$, ocurrió en el semestre 2020-A, que se llevó a cabo en modo virtual debido a la emergencia sanitaria provocada por COVID-19.

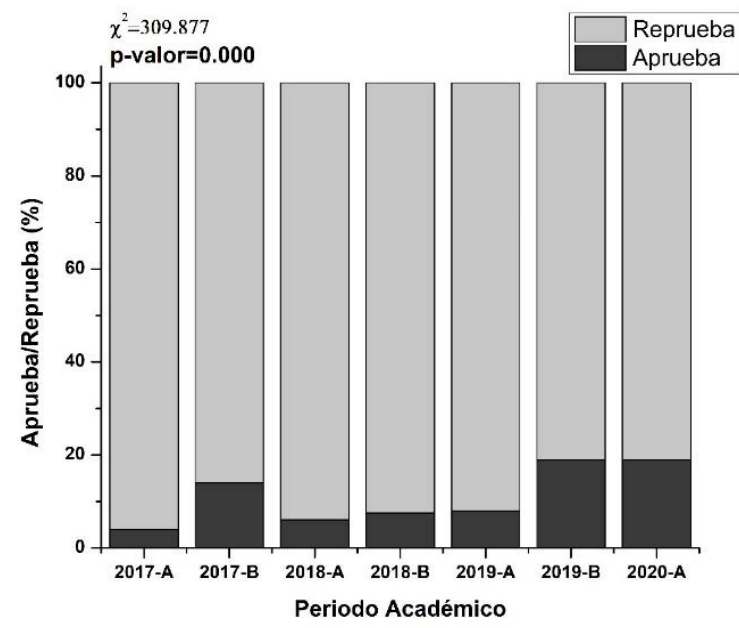

Figura 1. Porcentaje de aprobación y reprobación del curso de nivelación, según el tipo de estudio

En la figura 3 se muestra el gráfico de medias de las notas finales en las asignaturas de la asignatura de nivelación, para cada período académico.

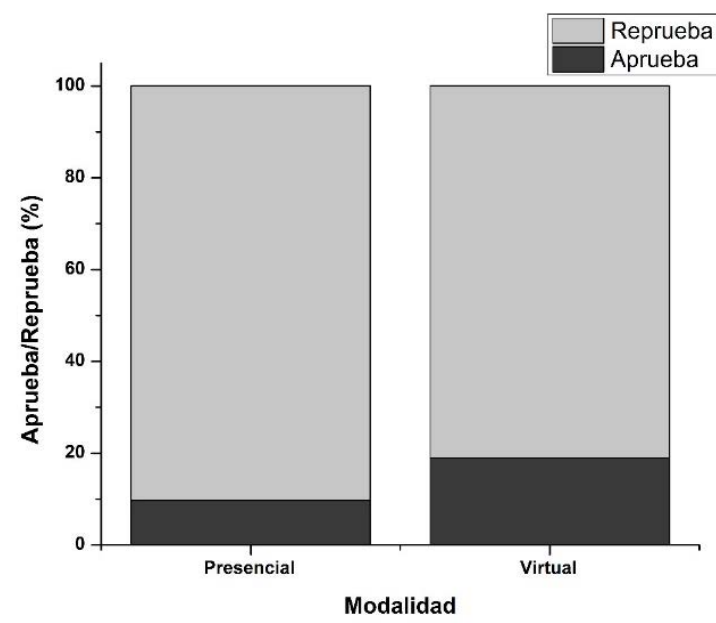

Figura 2. Porcentajes de aprobado y reprobado en el curso de nivelación. 


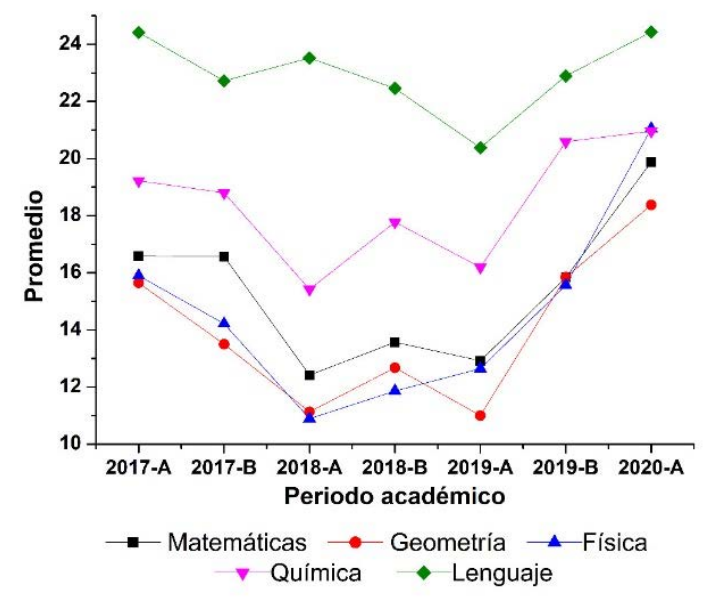

Figura 3. Gráfico de medias de las notas de las asignaturas de la asignatura de nivelación, en cada período académico.

Se observa que en el período 2020-A se obtuvo el mayor valor de la media para cada uno de los sujetos.

Estos hallazgos se comparan con la información presentada en la Tabla 2, donde se muestra que, efectivamente, existen diferencias significativas entre el desempeño de los estudiantes en cada uno de los períodos académicos.

Table 2. Resultados del ANOVA de un factor de la nota final de cada una de las asignaturas del curso de nivelación

\begin{tabular}{|c|c|c|c|}
\hline & & $\mathbf{F}$ & Sig. \\
\hline \multirow{3}{*}{ Matemática } & Entre grupos & 87.935 & .000 \\
\hline & $\begin{array}{c}\text { Dentro de } \\
\text { grupos }\end{array}$ & & \\
\hline & Total & & \\
\hline \multirow{3}{*}{ Geometría } & Entre grupos & 100.843 & .000 \\
\hline & $\begin{array}{c}\text { Dentro de } \\
\text { grupos }\end{array}$ & & \\
\hline & Total & & \\
\hline \multirow{3}{*}{ Física } & Entre grupos & 196.320 & .000 \\
\hline & $\begin{array}{c}\text { Dentro de } \\
\text { grupos }\end{array}$ & & \\
\hline & Total & & \\
\hline \multirow{3}{*}{ Química } & Entre grupos & 47.970 & .000 \\
\hline & $\begin{array}{l}\text { Dentro de } \\
\text { grupos }\end{array}$ & & \\
\hline & Total & & \\
\hline \multirow{3}{*}{ Lenguaje } & Entre grupos & 22,395 & .000 \\
\hline & $\begin{array}{c}\text { Dentro de } \\
\text { grupos }\end{array}$ & & \\
\hline & Total & & \\
\hline
\end{tabular}

Para determinar entre qué períodos académicos existieron diferencias significativas en las calificaciones finales, se realizó una prueba de Tukey, cuyos resultados se presentan en las tablas 3 a 7 .

Table 3. Resultados de la prueba de Tukey de la nota final en

\begin{tabular}{|c|c|c|c|c|}
\hline \multirow{2}{*}{$\begin{array}{l}\text { Inicio } \\
\text { del } \\
\text { Semestre }\end{array}$} & \multirow{2}{*}{$\mathbf{N}$} & \multicolumn{3}{|c|}{ Subconjunto para alfa $=0.05$} \\
\hline & & 1 & 2 & 3 \\
\hline 2018-A & 1343 & 12.4208 & & \\
\hline 2019-A & 1237 & 12.9224 & & \\
\hline 2018-B & 1411 & 13.5714 & & \\
\hline 2019-B & 1071 & & 15.8381 & \\
\hline 2017-B & 1369 & & 16.5733 & \\
\hline 2017-A & 1341 & & 16.5955 & \\
\hline 2020-A & 1324 & & & 19.8835 \\
\hline Sig. & & 0.063 & 0.489 & 1.000 \\
\hline
\end{tabular}

Table 4. Resultados de la prueba de Tukey de la nota final en Geometría

\begin{tabular}{|c|c|c|c|c|c|}
\hline \multirow{2}{*}{$\begin{array}{l}\text { Inicio del } \\
\text { Semestre }\end{array}$} & \multirow{2}{*}{$\mathbf{N}$} & \multicolumn{4}{|c|}{ Subconjunto para alfa $=\mathbf{0 . 0 5}$} \\
\hline & & 1 & 2 & 3 & 4 \\
\hline 2019-A & 1237 & 11.0011 & & & \\
\hline 2018-A & 1343 & 11.1308 & & & \\
\hline 2018-B & 1413 & & 12.6770 & & \\
\hline 2017-B & 1373 & & 13.4994 & & \\
\hline 2017-A & 1412 & & & 15.6482 & \\
\hline 2019-B & 1071 & & & 15.8486 & \\
\hline $2020-\mathrm{A}$ & 1324 & & & & 18.3790 \\
\hline Sig. & & 1.000 & .327 & .999 & 1.000 \\
\hline
\end{tabular}

Table 5. Resultados de la prueba de Tukey de la nota final en Física

\begin{tabular}{|c|c|c|c|c|c|c|}
\hline \multirow{2}{*}{$\begin{array}{c}\text { Inicio } \\
\text { del } \\
\text { Semestre } \\
\end{array}$} & \multirow{2}{*}{$\mathbf{N}$} & \multicolumn{5}{|c|}{ Subconjunto para alfa $=0.05$} \\
\hline & & 1 & 2 & 3 & 4 & 5 \\
\hline 2018-A & 1343 & 10.8926 & & & & \\
\hline 2018-B & 1413 & 11.8604 & 11.8604 & & & \\
\hline 2019-A & 1237 & & 12.6453 & & & \\
\hline 2017-B & 1373 & & & 14.2200 & & \\
\hline 2019-B & 1071 & & & & 15.5686 & \\
\hline 2017-A & 1436 & & & & 15.8993 & \\
\hline 2020-A & 1324 & & & & & 21.0524 \\
\hline Sig. & & .078 & .264 & 1.000 & .964 & 1.000 \\
\hline
\end{tabular}


Table 6. Resultados de la prueba de Tukey de la nota final en Química

\begin{tabular}{|c|c|c|c|c|c|}
\hline \multirow{2}{*}{$\begin{array}{c}\begin{array}{c}\text { Inicio } \\
\text { del } \\
\text { Semestre }\end{array} \\
\end{array}$} & \multirow{2}{*}{$\mathbf{N}$} & \multicolumn{4}{|c|}{ Subconjunto para alfa $=0.05$} \\
\hline & & 1 & 2 & 3 & 4 \\
\hline 2018-A & 1344 & 15.4243 & & & \\
\hline 2019-A & 1237 & 16.1968 & & & \\
\hline 2018-B & 1413 & & 17.7748 & & \\
\hline 2017-B & 1373 & & 18.8024 & 18.8024 & \\
\hline 2017-A & 1193 & & & 19.2276 & \\
\hline 2019-B & 1071 & & & & 20.5929 \\
\hline 2020-A & 1323 & & & & 20.9610 \\
\hline Sig. & & .542 & .196 & .955 & .978 \\
\hline
\end{tabular}

Table 7. Resultados de la prueba de Tukey de la nota final en Lenguaje

\begin{tabular}{|c|c|c|c|c|}
\hline \multirow{2}{*}{$\begin{array}{c}\text { Inicio } \\
\text { del } \\
\text { Semestre }\end{array}$} & \multirow[t]{2}{*}{$\mathbf{N}$} & \multicolumn{3}{|c|}{$\begin{array}{c}\text { Subconjunto para alfa }= \\
0.05\end{array}$} \\
\hline & & 1 & 2 & 3 \\
\hline 2019-A & 1237 & 20.3832 & & \\
\hline 2018-B & 1381 & & 22.4627 & \\
\hline 2017-B & 1371 & & 22.7225 & \\
\hline 2019-B & 1071 & & 22.8960 & \\
\hline 2018-A & 1343 & & 23.5252 & 23.5252 \\
\hline 2017-A & 960 & & & 24.4170 \\
\hline 2020-A & 1321 & & & 24.4344 \\
\hline Sig. & & 1.000 & .134 & .295 \\
\hline
\end{tabular}

\section{CONCLUSIONES}

Entre las medidas adoptadas por las universidades, a nivel mundial, se observa que muchas han adoptado mecanismos para promover la educación virtual, como es el caso de la Universidad Pedagógica y Tecnológica de Colombia (UPTC), que promovió la conectividad académica desde los hogares de los estudiantes, permitiendo interactuar la comunicación con mayor facilidad y mejorando así la asimilación de los conocimientos impartidos por los docentes, quienes utilizaron herramientas de acuerdo a la necesidad. Por ello, esta institución considera que el encierro social significó un cambio en el estilo de vida de los estudiantes, el cual debe ser estudiado para determinar la influencia que tuvo el aislamiento en su desempeño académico y en su entorno familiar (Valdivieso et al., 2020).

En cuanto a los resultados del primer caso con estudiantes de pregrado, se ha potenciado el $50 \%$ de las competencias en la modalidad presencial y virtual. En el segundo caso con estudiantes de pregrado, las actividades de aprendizaje desarrolladas virtualmente recibieron una buena ponderación, luego de incorporar buenas prácticas de aprendizaje en la redacción de estas actividades. Los resultados de los dos casos confirman la hipótesis de que la educación virtual es un medio para mejorar tanto las habilidades como el aprendizaje en los estudiantes universitarios (Durán, 2015).

Otro estudio muestra resultados de efectividad de la docencia virtual impartida en las tres modalidades: online, offline y en el medio (a distancia, presencial y semipresencial), donde los estudiantes tuvieron un desempeño similar, lo que significa que el aprendizaje puede tener el mismo éxito en cualquiera de las tres modalidades, siempre que las técnicas y metodologías utilizadas por los docentes sean de calidad, sumadas a un proceso de acompañamiento académico adecuado y oportuno (Yen et al., 2018).

Asimismo, otra investigación muestra que una metodología de enseñanza secuencial, bien estructurada y organizada, además de la tecnología adecuada para el desarrollo de aulas virtuales, dan lugar a la construcción de conocimiento con nuevos contenidos durante los espacios de trabajo. Para lograrlo, se utilizaron herramientas tecnológicas que incentivaron la participación activa de los estudiantes, con un pensamiento más amplio que les permitió resolver problemas originados dentro de sus clases virtuales. De hecho, se muestra que el rendimiento académico tuvo altos logros y esto se atribuye a la interacción realizada en los espacios virtuales que los estudiantes pudieron experimentar, donde también tuvieron la oportunidad de desarrollar habilidades interpretativas de textos y contenidos, de cada una de las asignaturas, lo que implica la continuidad de métodos autónomos y colaborativos en el nuevo modelo de enseñanza-aprendizaje, utilizando las herramientas tecnológicas precisas para el correcto funcionamiento del sistema durante el tiempo de transición en salud que atraviesa el mundo (Durso et al., 2020).

Según los resultados de la investigación, en cuanto a la homogeneidad de las calificaciones obtenidas en la asignatura de Lenguaje y comunicación, en los alumnos de los cursos de nivelación en el período 2017-2020; Por la naturaleza de la asignatura pertenece a las ciencias sociales y por tanto no corresponde al grupo de ciencias exactas, lo que permite que los estudiantes tengan más opciones de respuesta a una evaluación, expresando con mayor fluidez los conceptos relacionados con la asignatura, para desarrollar pensamiento lógico y habilidades que contribuyen al fortalecimiento de la memoria, la expresión verbal, las actitudes y el comportamiento.

Esto se basa en algunos datos que muestran que los estudiantes logran competencias importantes dentro del proceso de aprendizaje, ya que permite el desarrollo de habilidades tanto cognitivas como lingǘsticas, las cuales se expresan dentro de un espacio de consenso para alcanzar la objetividad de la ciencia, pudiendo interpretar y argumentar los contenidos y textos con mayor libertad, a través del lenguaje oral y escrito. Asimismo, desde la teoría lógica de la investigación, los productos de la mente humana están influenciados por el lenguaje, ya que el ser humano puede alcanzar altos niveles de comprensión para el conocimiento subjetivo y objetivo, lo que significa que para mantener vivo el conocimiento, el uso del lenguaje en sus dos formas es importante (Martínes et al., 2013).

Si bien los resultados del desempeño académico para el período 2020-A, de la educación virtual, muestran calificaciones más altas en comparación con los períodos anteriores donde la educación fue presencial, esto no 
necesariamente indica que los estudiantes de ese período y modalidad han obtenido más conocimientos. Algunos autores coinciden en que el desempeño académico de los estudiantes universitarios no es un referente para la evaluación cognitiva en toda su magnitud, ya que este proceso provoca el etiquetado de ellos, los sujetos y los propios docentes, ubicándolos en un rango de evaluación que puede determinar la capacidad, efectividad y calidad del proceso de enseñanza-aprendizaje. Sin embargo, también se pueden presentar criterios de dificultad de las asignaturas, observándose en el caso contrario, que un alumno con bajo rendimiento reprueba y una asignatura con bajo promedio puede ser más importante en el plan de estudios. Por ello, mencionan que la calificación es un mecanismo de evaluación que requiere una verificación de las capacidades personales de los estudiantes y que en realidad un valor no expresa su nivel cognitivo, ya que la aplicación de un sistema de evaluación tradicional contrasta con la verdadera capacidad de estudiantes (Rojas Betancur \& González, 2009).

\section{REFERENCIAS}

Benítes, S., \& Cabrera, L. (2021). Impacto de la pandemia covid-19 en el rendimiento estudiantil a través del teleaprendizaje en estudiantes de la carrera de enfermería. [Universidad de Guayaquil]. http://repositorio.ug.edu.ec/bitstream/redug/53805/1/168 2-TESIS-BENÍTEZ-CABRERA- LIC. YOUDESLEY AVILA.pdf

CEPAL-UNESCO. (2020). La educación en tiempos de la pandemia de COVID-19. https://www.siteal.iiep.unesco.org/respuestas_educativas _covid_19.

CES., C. de E. S. (2019). Reglamento de Régimen académico. (Issue 111).

D’Amelio, A., Nardechia, L., \& Gisel Tari, J. (2020). Burnout y coronavirus: los efectos de la pandemia en estudiantes universitarios. Universidad Nacional de Córdova, Argentina. https://rdu.unc.edu.ar/handle/11086/16946

Durán, R. A. (2015). La Educación Virtual Universitaria como medio para mejorar las competencias genéricas y los aprendizajes a través de buenas prácticas.

Durso, G. :, Tomaghelli, E. ;, Varela, J., Bustichi, G.; \& Pollicina, L. ; (2020). APRENDER CON TECNOLOGÍA EN EPOCA DE PANDEMIA. http://sedici.unlp.edu.ar/handle/10915/107875

Martínes, A., Quintero, G., \& Ruiz, Y. (2013). La importancia del lenguaje en los procesos de aprendizaje. 57(1), 1730 .

Ordorika Imanol. (2020). Pandemia y educación superior.
RESU, Revista de La Educación Superior, 1-8. https://doi.org/10.36857/resu.2020.194.1120

Organización Mundial de la Salud. (2020, March 11). La OMS caracteriza a COVID-19 como una pandemia - OPS/OMS | Organización Panamericana de la Salud. Organización Mundial de La Salud. https:/www.paho.org/es/noticias/11-3-2020-omscaracteriza-covid-19-como-pandemia

Parada, M., \& Pérez, C. (2014). Relación del engagement académico con características académicas y socioafectivas en estudiantes Relation between Academic Engagement and academic and affective characteristics of dental students. Educación Médica Superior, 28(2), 199215. http://scielo.sld.cu

Paredes Chacín, A. J., Inciarte Gonzáles, A., \& Walles Peñaloza, D. (2020). Educación superior e investigación en Latinoamérica: Transición al uso de tecnologías digitales por Covid-19. 98-117. https://orcid.org/00000002-3682-6061

Rojas Betancur, M., \& González, D. C. (2009). Rendimiento y calificación, dos aspectos problemáticos de la evaluación en la universidad. Revista Virtual Universidad Católica Del Norte. http://revistavirtual.ucn.edu.co/],

Universia.net. (2020). Cómo estudiar medicina a distancia. https://www.universia.net/es/actualidad/orientacionacademica/como-estudiar-medicina-distancia1161337.html

Universidad Johns Hopkins. (2021, April 25). COVID-19 Dashboard by the Center for Systems Science and Engineering (CSSE) at Johns Hopkins University (JHU). https://www.arcgis.com/apps/opsdashboard/index.html\#/ bda7594740fd40299423467b48e9ecf6

Valdivieso, M. A., Burbano, V. M., \& Burbano, Á. S. (2020). Percepción de estudiantes universitarios colombianos sobre el efecto del confinamiento por el coronavirus, y su rendimiento académico Perception of Colombian university students on the effect of confinement by the coronavirus, and their academic performan. Revista Espacios, 41. https://doi.org/10.48082/espaciosa20v41n $42 \mathrm{p} 23$

Yen, S. C., Lo, Y., Lee, A., \& Enriquez, J. M. (2018). Learning online, offline, and in-between: comparing student academic outcomes and course satisfaction in face-toface, online, and blended teaching modalities. Education and Information Technologies, 23(5), 2141-2153. https://doi.org/10.1007/s10639-018-9707-5 